\title{
Neonatal vitamin A supplementation for prevention of mortality and morbidity in infancy: systematic review of randomised controlled trials
}

\author{
Siddhartha Gogia, attending consultant, Harshpal Singh Sachdev, senior consultant
}

Department of Paediatrics and Clinical Epidemiology, Sitaram Bhartia Institute of Science and Research, B-16 Qutab Institutional Area, New Delhi 110016, India Correspondence to: HSSachdev, E6/12 Vasant Vihar, New Delhi 110057, India

hpssachdev@gmail.com

Cite this as: BMJ 2009;338:b919 doi:10.1136/bmj.b919

\section{ABSTRACT}

Objective To evaluate the effect of neonatal vitamin A supplementation on infant mortality, morbidity and early adverse effects.

Design Systematic review, meta-analysis, and metaregression of randomised controlled trials.

Data sources Electronic databases and hand search of reviews; abstracts and proceedings of conferences.

Review methods Randomised or quasi-randomised or cluster randomised, placebo controlled trials evaluating the effect of prophylactic, neonatal ( $<1$ month)

supplementation with synthetic vitamin $A$ on mortality or morbidity within infancy ( $<1$ year), and early adverse effects ( $\leq 7$ days).

Results The six included trials were from developing countries. There was no convincing evidence of a reduced risk of mortality during infancy (relative risk 0.92, 95\% confidence interval 0.75 to $1.12, \mathrm{P}=0.393$ random effect; $\left.\mathrm{I}^{2}=54.1 \%\right)$ or of an increase in early adverse effects including bulging fontanelle (1.16, 0.81 to $1.65, \mathrm{P}=0.418$; $\left.\mathrm{I}^{2}=65.3 \%\right)$. No variable emerged as a significant predictor of mortality, but data for important risk groups (high maternal night blindness prevalence and low birth weights) were restricted. Limited data (from one to four trials) did not indicate a reduced risk of mortality during the neonatal period $\left(0.90,0.75\right.$ to $\left.1.08, P=0.270 ; I^{2}=0 \%\right)$, cause specific mortality, common morbidities (diarrhoea and others), and admission to hospital. There was, however, evidence of an increased risk of acute respiratory infection and a reduced risk of clinic visits. Conclusions There is no convincing evidence of a reduced risk of mortality and possibly morbidity or of increased early adverse effects after neonatal supplementation with vitamin $A$. There is thus no justification for initiating such supplementation as a public health intervention in developing countries for reducing infant mortality and morbidity.

\section{INTRODUCTION}

Prophylactic vitamin A supplementation for children aged 1-4 is considered to be an effective intervention for improving child survival in developing countries. ${ }^{1}$ Three previous systematic reviews ${ }^{2-4}$ have shown associated reductions of $23-30 \%$ in childhood mortality.
Similar survival benefits have also been reported in some trials that included infants aged 6-11 months. ${ }^{5-7}$ There is, however, no evidence of a reduction in mortality at 1-6 months..$^{8-11 \mathrm{w} 1}$ A recent review concluded that neonatal vitamin A supplementation is associated with $20 \%$ reduction in mortality in babies under 6 months and included it as a core public health intervention for the Asian region. ${ }^{12}$ These findings have been contested because relevant but negative data were ignored. ${ }^{13}$ To provide updated evidence to inform policy for an annual birth cohort of over 50 million infants, we conducted a systematic review of randomised controlled trials to evaluate the effect of prophylactic neonatal supplementation with synthetic vitamin A on mortality and morbidity in infancy and early adverse effects.

\section{METHODS}

We evaluated the effect of prophylactic supplementation with synthetic vitamin A in the neonatal period $<<$ 1 month), irrespective of maternal antenatal or postnatal vitamin A supplementation status, on mortality and morbidity in infancy ( $<1$ year), and early adverse effects (within one week after the intervention).

We had planned to carry out eight prespecified subgroup analyses for the all cause mortality within 1 year:

- Cumulative vitamin A dose received till the age of 1 month to examine the possibility of a doseresponse relation through a stratified comparison of low ( $\leq 50000 \mathrm{IU}) v$ high $(>50000 \mathrm{IU})$ doses

- Number of vitamin A doses received to examine the possibility of a relation to dosing frequency through a stratified comparison of single $v$ multiple $(\geq 2)$ doses

- Maternal postpartum vitamin A supplementation to examine the possibility of an interactive effect through a stratified comparison of present $(\geq 30 \%$ received simultaneous maternal postpartum supplementation) $v$ absent $(<30 \%$ received simultaneous maternal postpartum supplementation)

- Baseline maternal vitamin A status to examine the possibility of a greater response in deficient participants through a stratified comparison of 
prevalence of maternal night blindness of $<5 \%$ (low) $v \geq 5 \%$ (high), and mean maternal antenatal or postpartum serum retinol concentrations of $\geq 1.1 \mu \mathrm{mol} / 1$ (low maternal deficiency) $v<1.1$ $\mu \mathrm{mol} / \mathrm{l}$ (high maternal deficiency)

- Birth weight of the offspring to examine the possibility of a greater response in more deficient low birthweight newborns through a comparison of mortality estimates among newborns with birth weight $<2500$ g $v \geq 2500$ g (normal birth weight)

- Infant mortality rate in the placebo group to examine the possibility of a greater response with higher baseline mortality through a stratified comparison of lower $v$ upper half (median) of infant mortality rate in included trials

- Follow-up age to examine the possibility of a greater response in the first half of infancy through a stratified comparison of follow-up age $\leq 6$ months $v>6$ months and

- Development status of the trial area to examine the possibility of a greater response in high risk populations through a stratified comparison of developing $v$ developed countries.

The trial sites were classified as developing countries if they were categorised as low or medium in the human development index. ${ }^{14}$

\section{Criteria for inclusion}

Types of trials - We included randomised or quasi-randomised or cluster randomised, placebo controlled trials regardless of publication status and language, that evaluated the effect of prophylactic supplementation with synthetic vitamin A initiated in the neonatal period ( $<1$ month), irrespective of maternal antenatal or postnatal vitamin A supplementation status, on mortality or morbidity in infancy $(<1$ year), or early adverse effects (within a week after the intervention). Trials using a factorial design with multiple intervention groups were eligible for inclusion.

Types of participants - Participants were apparently healthy infants in whom prophylactic, synthetic vitamin A supplementation was initiated in the neonatal period ( $<1$ month), irrespective of maternal antenatal or postnatal vitamin A supplementation status. We excluded trials conducted on selected subgroups of infants, such as those who were very low birth weight $(<1500 \mathrm{~g})$, HIV positive, born to HIV positive mothers, or sick or admitted to hospital, as these have been examined elsewhere. ${ }^{1516}$

Types of intervention - Synthetic oral vitamin A supplementation initiated in the neonatal period $<<$ 1 month), irrespective of maternal antenatal or postnatal vitamin A supplementation status, was compared with placebo administered to the infant and either placebo or no supplementation in the mother. If such a comparison group was not available for the motherinfant, the intervention group was compared with the group in which the infant had received placebo while the mother had received supplementation identical to the intervention group. We included trials providing additional interventions if the only difference between the treatment arms was synthetic oral vitamin A supplementation. We excluded trials supplementing vitamin A rich foods or $\beta$ carotene.

\section{Types of outcome measures}

Primary-We examined all cause mortality in the child at two time points: during infancy, in the period between initiation of intervention and the last followup within the age of 1 year; and during the neonatal period between initiation of intervention and the last follow-up within the age of 1 month.

Secondary-In the period between initiation of the intervention and the last follow-up within the age of 1 year we measured cause specific mortality because of diarrhoea, acute respiratory infections, and causes other than these (as defined by the authors, irrespective of ascribing a single or multiple causes of death); morbidities because of diarrhoea, acute respiratory infection or respiratory difficulty, cough or running nose, ear infection, fever, and vomiting (as defined by the authors); and severity of morbidities as assessed by clinic or hospital visits and admissions to hospital (as defined by the authors). We also measured early adverse effects including bulging fontanelle, vomiting, irritability, diarrhoea, and fever (as defined by the authors) within one week after the intervention.

\section{Searches}

We searched computerised bibliographic medical databases, including Medline, CENTRAL, Embase, IBIDS, CINAHL, and HealthSTAR and the clinical trials website (www.clinicaltrials.gov) up to 5 July 2008 with no language restrictions. The broad search strategy used for Medline was: ("vitamin A" OR retinol) AND (Clinical Trial[ptyp] OR Meta-Analysis [ptyp] OR Randomized Controlled Trial[ptyp] OR Review[ptyp] OR Controlled Clinical Trial[ptyp] OR Journal Article[ptyp]) AND (infant[MeSH]). We scanned the title and abstract of the trials identified to exclude those that were obviously irrelevant, retrieved the full text of the remaining trials, and identified relevant articles. We also carried out a lateral search using the related articles link in PubMed for the articles initially included from the search strategy. We reviewed the reference lists of identified articles and hand searched reviews and abstracts and proceedings of international micronutrient conferences. We contacted donor agencies, "experts," and authors of recent vitamin A supplementation trials to identify any additional or ongoing trials.

\section{Assessment of quality of methods}

We assessed quality of the identified trials by the standard criteria with respect to allocation concealment, follow-up and blinding. ${ }^{17}$ The scores assigned were as adequate, unclear, inadequate, and not used for randomisation; adequate, unclear, inadequate, and not used for allocation concealment; <3\%, 3-9.9\%, 10-19.9\%, and $\geq 20 \%$ for attrition (percentage of participants 
excluded); and double blinding, single blinding, no blinding, and unclear for blinding. Our prespecified sensitivity analyses on the basis of trial quality were allocation concealment (adequate $v$ others), attrition $(<10 \% \quad v \geq 10 \%$ ), and blinding (double blinding $v$ others).

\section{Data abstraction}

We extracted data in duplicate on a pre-tested questionnaire and contacted authors for additional information, if required. For vitamin A supplementation we collected information on age, form, route, dose, and frequency. We also recorded data on various aspects including the trial site, baseline characteristics of participants, inclusion and exclusion criteria, cointerventions, compliance monitoring, intention to treat analysis, and cluster adjustment.

In factorial trials and in multi-arm designs yielding two or more intervention groups (different dose or administration regimens, or maternal supplementation status) and a single control group, we pooled the data in the intervention groups, including the variation in the intervention characteristic, and compared these against the single control group to prevent unit of analysis error. $^{18}$

\section{Statistical analysis}

For computing the summary relative risk, we used relative risks and 95\% confidence intervals or standard errors from individual studies. In a hierarchical pattern, we gave preference to the relative risk stated by authors and rechecked the calculations from the stated numbers. If the relative risk was not stated, we calculated it with the following preference order for the denominator: stated child years, numbers with definite outcome known till completion of intervention period, or number randomised. In trials that did not report intention to treat analysis but had relevant data available, we reconstructed intention to treat estimates. For cluster randomised trials, we used the stated cluster adjusted relative risk and 95\% confidence interval, irrespective of the method used. In the absence of this information, we sought raw data from authors to calculate the design effect. If these were not available, we intended to recalculate the relative risks for sensitivity analysis using a design effect inflation of $\mathrm{SE}^{19}$ by the pooled estimate based on other cluster randomised trials. If there were no events (or all events) in both groups the trial provided no information about relative probability of the event and was automatically omitted from the meta-analysis. ${ }^{19}$

Data entry and initial analysis was performed with SPSS (version 13.0) software and meta-analysis and meta-regression with user written programs on Stata (version 9.2) software. We evaluated the presence of bias in the extracted data using funnel plots. ${ }^{20} \mathrm{We}$ also carried out formal statistical tests for funnel plot asymmetry - namely, the methods of Begg and Egger -with the user written "metabias" command in Stata (version 9.2) software. $^{2122}$ Pooled estimates (relative risks with $95 \%$ confidence intervals) of the evaluated

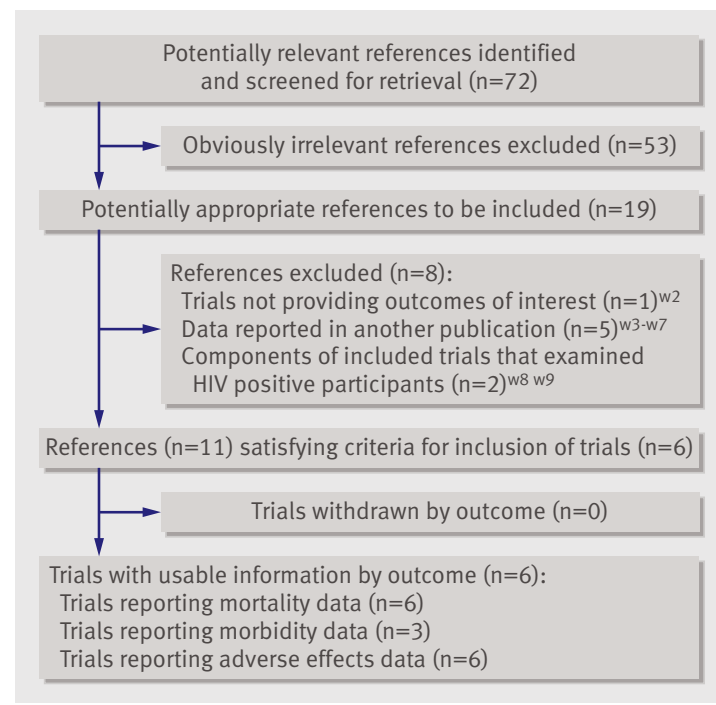

Fig 1| Trial flow for selection of randomised controlled trials for inclusion in meta-analysis

outcome measures were calculated by the generic inverse variance method by the user written "metan" command in Stata (version 9.2) software. ${ }^{2123}$ The natural logarithm converted values of the individual study relative risks and their SEs were used for computing the pooled estimates as recommended. ${ }^{23}$ These pooled estimates were computed with both fixed effects and random effects model assumptions and expressed in an exponential form. This program also computes the formal tests of heterogeneity-namely, the statistic Cochran $Q$ and $\mathrm{I}^{2}$ (variation in pooled estimate attributable to heterogeneity). ${ }^{24}$

We carried out sensitivity and subgroup analyses (specified above) for the primary outcome (all cause mortality during infancy) by disaggregating results with the user written "metan" command ("by option") in Stata (version 9.2) software. ${ }^{2123}$ A separate analysis was done for those trials providing disaggregated information for mortality for low birthweight and non-low birthweight infants. We could not carry out a subgroup analysis of baseline mortality in the placebo group because we could not get a uniform measure across the included trials. We explored the contribution of these variables to heterogeneity by meta-regression using the "metareg" command in Stata (version 9.2) software with the restricted maximum likelihood option. ${ }^{25}$

\section{RESULTS}

Trial flow

We identified 19 potentially eligible references. ${ }^{\text {w1-w19 }}$ We excluded eight references (fig 1). ${ }^{\text {w2-w9 }}$ The 11 remaining references provided data on six trials that satisfied the inclusion criteria; six trials provided mortality data, three had relevant data on morbidity, and six provided data on adverse effects.

\section{Study characteristics}

The baseline characteristics of the included trials and the notable general and individual study specific 
features in relation to data abstraction are in tables $\mathrm{A}$ and $\mathrm{B}$ and appendix 1 on bmj.com.

\section{Quantitative data synthesis Mortality during infancy}

The six trials were conducted in developing countries (four in Asia and two in Africa). Two cluster randomised trials were included with design adjusted results. ${ }^{\mathrm{w} 1 \mathrm{w} 14}$ All trials were double blind with adequate allocation concealment, and loss to follow-up was below $10 \%$ in four trials. Three trials followed up participants up to 6 months of age. Two trials gave simultaneous maternal postpartum vitamin A supplementation $(\geq 30 \%$ mothers in the intervention arm). In all trials the cumulative dose of vitamin A was $\leq 50000 \mathrm{IU}$, given as a single dose in five trials and as two doses in one trial. Information on prevalence of maternal night blindness was available in only three trials; of these one recorded a prevalence $<5 \%$. In the three trials that provided this information mean maternal serum retinol concentration in the placebo group was $\geq 1.1 \mu \mathrm{mol} / \mathrm{l}$. Three trials presented results separately for low birthweight and non-low birthweight infants.

The funnel plot was symmetrical, suggesting the absence of publication bias, which we confirmed using Egger's (weighted regression) method ( $\mathrm{P}=0.931$ for bias) and Begg's (rank correlation) method (continuity corrected $\mathrm{P}=1.0)$. There was no convincing evidence of a reduced risk of mortality during infancy; the pooled relative risk was $0.92(95 \%$ confidence interval 0.75 to $\left.1.12, \mathrm{P}=0.393 ; \mathrm{I}^{2}=54.1 \%, \mathrm{P}=0.053\right)$ by random effects model (table 1 and fig 2). Prespecified sensitivity, subgroup, and meta-regression analyses did not identify a consistently significant predictor of mortality response (tables 1 and 2). Stratified analysis of limited

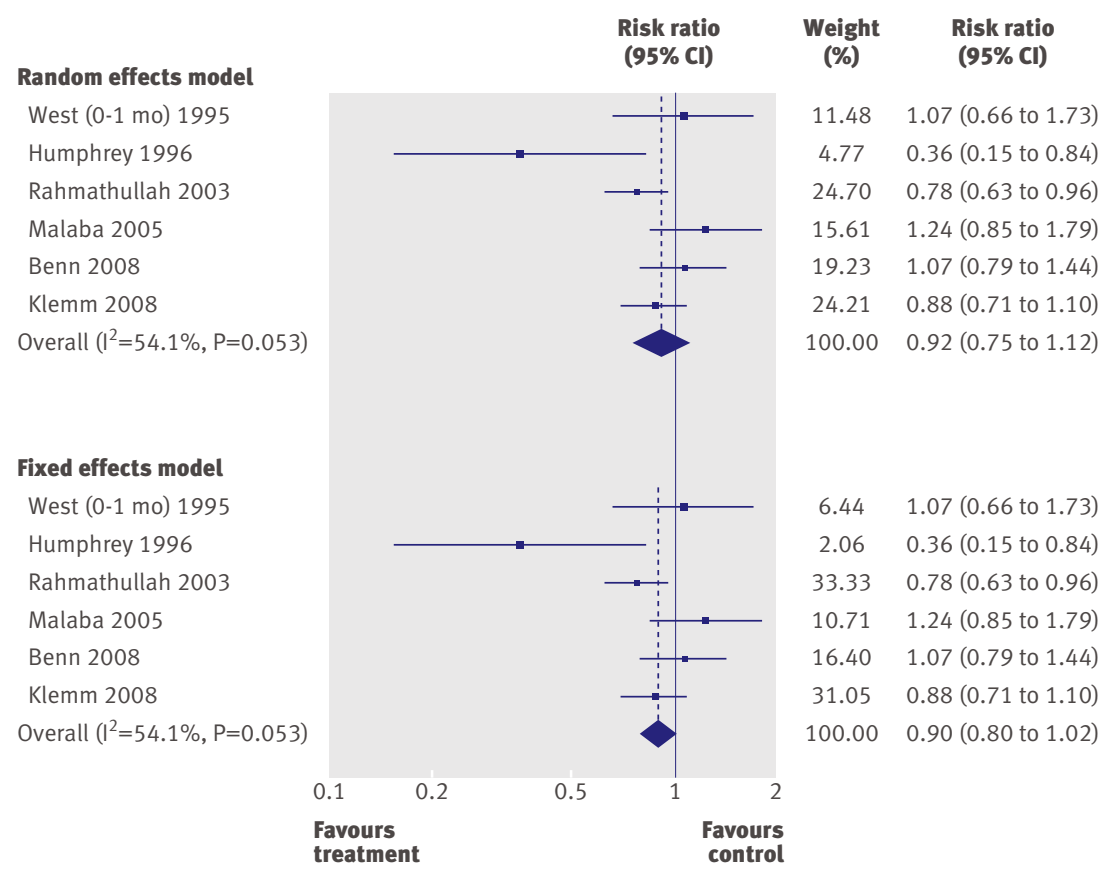

Fig 2 | Forest plots for relative risk of mortality during infancy data (three trials), however, suggested a greater response with $\geq 5 \%$ prevalence of maternal night blindness.

\section{Mortality during neonatal period}

We pooled data from three trials, two from Asia ${ }^{\text {w12 w14 }}$ and one from Africa. ${ }^{\text {w11 }}$ Data from the African trial pertained to the first seven days of life only. In the factorial design study, mothers in placebo group received supplementation (synthetic vitamin A or $\beta$ carotene) identical to the intervention group. ${ }^{\text {w14 }}$ There was no convincing evidence of a reduced risk of mortality during the neonatal period; pooled relative risk for mortality was 0.90 ( 0.75 to $\left.1.08, \mathrm{P}=0.270 ; \mathrm{I}^{2}=0 \%, \mathrm{P}=0.834\right)$ by random and fixed effects models.

\section{Cause specific mortality}

We pooled data ascertained by verbal autopsy from

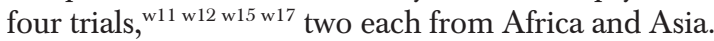
There was no convincing evidence of a reduced risk of mortality from respiratory, diarrhoeal, or other causes by random effects model (table 3).

\section{Morbidities and their severity}

We pooled data from three trials, two from Asia ${ }^{\text {w12 w18 }}$ and one from Africa. ${ }^{\text {w16 }}$ All these trials were double blind with adequate allocation concealment and $<10 \%$ loss to follow-up in two. ${ }^{\text {w16 w18 }}$ Two trials used a single intervention dose, ${ }^{\text {w12 }}{ }^{\text {w16 }}$ and in two the follow-up age was $>6$ months. ${ }^{\text {w12 w16 }}$ There was no evidence of a reduced risk of diarrhoea, cough or running nose, ear infection, fever, or vomiting (table 3). There was an increased risk of acute respiratory infection or respiratory difficulty $\left(1.11,1.02,1.21 ; \mathrm{P}=0.015 ; \mathrm{I}^{2}=0\right.$, two trials). There was a reduced risk of clinic visits $(0.81$, $0.72,0.91 ; \mathrm{P}=0.001 ; \mathrm{I}^{2}=0$, two trials) and no evidence of a reduced risk of admission to hospital $(0.75,0.26$, $2.16 ; \mathrm{P}=0.593$; one trial).

\section{Early adverse effects}

We pooled data from six trials (four from Asia ${ }^{\text {w10 w14 w18 }}$ ${ }^{\text {w19 }}$ and two from Africa ${ }^{\text {w13 w16) }}$; one trial did not record any episode of bulging fontanelle $\mathrm{e}^{\mathrm{w} 17}$ and one did not record any irritability ${ }^{\mathrm{w} 19}$ in either group. Only two trials were cluster randomised, but their relative risks for adverse effects were unadjusted for design effect. ${ }^{\text {w14 }}$

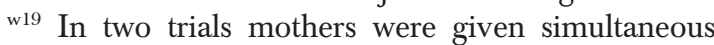
maternal postpartum vitamin A supplementation. ${ }^{\text {w13 }}$ ${ }^{\text {w14 }}$ In all trials infants received $\leq 50000$ IU vitamin A. In two trials physicians recorded bulging of fontanelle. ${ }^{\text {w10 w16 }}$ In one trial about two thirds of the mothers of infants in the placebo group received either synthetic vitamin A or $\beta$ carotene. ${ }^{\text {w14 }}$ There was no convincing evidence of an increased risk of bulging fontanelle, vomiting, irritability, diarrhoea, or fever (table 3).

\section{DISCUSSION}

We found no convincing evidence of a reduced risk of all cause mortality during infancy or of an increased risk of early adverse effects, including 
bulging fontanelle, after neonatal supplementation with synthetic vitamin A. Limited data did not indicate a reduced risk of mortality during the neonatal period, cause specific mortality, morbidities (diarrhoea and others), and admission to hospital. There was, however, evidence of an increased risk of acute respiratory infection and a reduced risk of clinic visits. No variable emerged as a consistent significant predictor of mortality during infancy but the data for important risk groups (high prevalence of maternal night blindness and low birth weight) were quite restricted.

\section{Strengths and limitations of analyses}

In our systematic review we incorporated relevant sensitivity and meta-regression analyses. Analysis of six trials, though admittedly not robust proof, indicated no formal evidence of publication bias. Cluster and individual randomised trials were appropriately combined by design effect correction for the primary outcome. The main conclusion regarding all cause mortality remained stable over a large spectrum of sensitivity analyses.

Our study has some specific limitations. All the trials we included were conducted in developing countries, which limits the generalisation of findings. We had limited data on high risk groups (maternal night blindness $\geq 5 \%$ and low birthweight infants). In the two cluster randomised trials, adverse effects were unadjusted for design effect. Duration of follow-up was variable, which precluded constitution of a uniform measure to explore baseline mortality as a predictor. Finally, we used multiple subgroup and meta-regression analyses, which increased the possibility of false positive results.
Any identified significant predictor should therefore be considered only as exploratory.

\section{Inclusion and exclusion criteria}

Although we intended to exclude non-placebo but controlled trials to obviate bias caused by the "Hawthorne effect," ${ }^{26-29}$ we did not find any such trials. We excluded trials in which participants were HIV positive or neonates born to HIV positive mothers to factor for potential effect modification by an immunosuppressive condition. In some settings, however, it would be impossible to distinguish such participants from HIV negative participants. There was no convincing evidence of a reduced risk of mortality during infancy with data from HIV positive mothers included $\left(0.96,0.80,1.15 ; \mathrm{P}=0.674 ; \mathrm{I}^{2}=65 \%\right) .{ }^{\mathrm{w} 9} \mathrm{We}$ restricted age at intervention to the first month of life because of pragmatic considerations. As the terms newborn and neonate are used interchangeably to denote age below 1 month, an alternative time frame of providing "vitamin A supplements to newborns within the first few days of life" ${ }^{30}$ will create ambiguity. Furthermore, it would sacrifice data from relevant trial(s), which do not provide outcomes stratified by exact age within 1 month. The option of including synthetic vitamin A as a component of the safe delivery kit could not be examined because the included trials were not specifically designed to administer the drug at birth (immediately after umbilical cord separation). Nevertheless, the conclusion regarding mortality during infancy remained stable when we analysed only dosing within narrow time windows after birth from the available data in the five trials irrespective of maternal vitamin

\begin{tabular}{|c|c|c|c|c|c|}
\hline \multirow[b]{2}{*}{ Stratification variable* } & \multirow[b]{2}{*}{ No of trials } & \multicolumn{2}{|c|}{ RR $(95 \% \mathrm{Cl}) ; \mathrm{P}$ value } & \multirow{2}{*}{$\begin{array}{l}\text { Tests for heterogeneity I2 } \\
\text { (\%); Q (P value) }\end{array}$} & \multirow{2}{*}{$\begin{array}{l}\text { P value for heterogeneity in } \\
\text { subgroups }\end{array}$} \\
\hline & & Random effects model & Fixed effects model & & \\
\hline Overall & 6 & 0.92 (0.75 to 1.12$) ; 0.393$ & 0.90 (0.80 to 1.02 ); 0.090 & $54.1 ; 10.90(0.053)$ & NA \\
\hline \multicolumn{6}{|l|}{ Attrition: } \\
\hline$\ll 10 \%$ & 4 & 0.89 (0.77 to 1.03$) ; 0.119$ & 0.89 (0.78 to 1.01$) ; 0.065$ & $14.8 ; 3.52(0.318)$ & \\
\hline$\geq 10 \%$ & 2 & 0.71 (0.21 to 2.36$) ; 0.576$ & 1.01 (0.72 to 1.42$) ; 0.939$ & $85.4 ; 6.84(0.009)$ & 0.465 \\
\hline \multicolumn{6}{|l|}{ Follow-up age (months): } \\
\hline$\leq 6$ & 3 & 0.85 ( 0.73 to 0.98$) ; 0.024$ & 0.85 (0.73 to 0.98$) ; 0.024$ & $0.0 ; 1.63(0.444)$ & \\
\hline$>6$ & 3 & 0.92 (0.57 to 1.48$) ; 0.715$ & 1.05 (0.83 to 1.31$) ; 0.703$ & $71.0 ; 6.9(0.032)$ & 0.123 \\
\hline \multicolumn{6}{|c|}{ Maternal postpartum vitamin A supplementation ( $230 \%$ mothers): } \\
\hline No & 4 & 0.85 (0.63 to 1.16$) ; 0.305$ & 0.86 (0.73 to 1.01$) ; 0.062$ & $61.2 ; 7.72(0.052)$ & \\
\hline Yes & 2 & 1.01 (0.73 to 1.40$) ; 0.961$ & 0.96 (0.80 to 1.16$) ; 0.676$ & $58.1 ; 2.39(0.122)$ & 0.374 \\
\hline \multicolumn{6}{|c|}{ No of vitamin A doses received: } \\
\hline Single & 5 & 0.96 (0.76 to 1.23$) ; 0.767$ & 0.97 (0.83 to 1.12$) ; 0.659$ & $51.4 ; 8.23(0.083)$ & \\
\hline Multiple & 1 & 0.78 (0.63 to 0.96$) ; 0.021$ & 0.78 (0.63 to 0.96$) ; 0.021$ & NA & 0.102 \\
\hline \multicolumn{6}{|l|}{ Maternal night blindness: } \\
\hline$<5 \%$ & 1 & 1.24 (0.85 to 1.79 ); 0.262 & 1.24 (0.85 to 1.79$) ; 0.262$ & NA & \\
\hline$\geq 5 \%$ & 2 & 0.83 (0.71 to 0.96$) ; 0.014$ & 0.83 (0.71 to 0.96$) ; 0.014$ & $0.0 ; 0.61(0.433)$ & 0.049 \\
\hline \multicolumn{6}{|l|}{ Birth weight specific $†$ : } \\
\hline Low birth weight & 3 & 0.76 (0.58 to 1.01$) ; 0.056$ & 0.80 (0.69 to 0.93$) ; 0.003$ & $52.7 ; 4.23(0.121)$ & 0.380 \\
\hline Other & 3 & 0.78 ( 0.43 to 1.40$) ; 0.399$ & $0.92(0.70$ to 1.20$) ; 0.514$ & $63.7 ; 5.50(0.064)$ & \\
\hline
\end{tabular}


A supplementation status in the control groups. ${ }^{\text {w11 w12 }}$ w14 w15 w17 The pooled estimate (random effects model) was $0.89\left(0.73,1.09 ; \mathrm{P}=0.256 ; \mathrm{I}^{2}=56 \%\right)$ for dosing during the first 48 hours after birth and $0.88(0.73,1.07$; $\mathrm{P}=0.196 ; \mathrm{I}^{2}=53 \%$ ) for dosing during the first seven days of life. To evaluate cause specific mortality, we decided to pool data from trials reporting a single or multiple cause of death, with the underlying philosophy that the assessed cause had contributed to mortality.

\section{Choice of comparison group}

A key issue for data abstraction in multi-arm and factorial design trials is the choice of the comparison group. For several reasons our control group comprised neonates who were given placebo and whose mothers had received either placebo or no supplementation. The most satisfactory comparison for policy should replicate the envisaged intervention, and currently simultaneous neonatal and maternal supplementation is not under consideration. Also, vitamin A transferred through breast milk might interact with neonatal intervention. Postpartum vitamin A supplementation to $\mathrm{HIV}$ positive mothers whose infants remained polymerase chain reaction-negative at 6 weeks $^{\mathrm{w} 9}$ was associated with increased mortality by age 2 (hazard ratio $1.82,0.99$ to $3.31 ; \mathrm{P}=0.05$ ). Other trials of antenatal and/or postnatal maternal supplementation $^{31 \mathrm{w15}}$ also documented an increased risk of mortality for offspring (relative risks 1.05 and $1.26 ; \mathrm{P}>0.05)$. Including maternal supplementation in a control group could thus conceivably inflate survival benefit by increasing mortality in the neonatal placebo group. Relevant factorial designed trials were powered only to detect effect sizes pooled across the various subgroups (maternal supplementation arms). ${ }^{\text {w14 w15 }}$ Such pooling is usually justified by post hoc subgroup analyses that show no significant interaction between maternal and newborn supplementation. These analyses, however, are underpowered to reveal realistic interactions; the power was only $10 \%$ to detect an interaction term (0.88) equivalent to the observed effect size in the maternal placebo subgroup in one study. ${ }^{\text {w14 }}$ Trials with $80 \%$ power for the overall effect have only $29 \%$ power to detect an interaction effect of the same magnitude, and even less power for

Table 2 | Meta-regression analyses (univariate analysis*) of all cause infant mortality after neonatal vitamin A supplementation

\begin{tabular}{|c|c|c|}
\hline Study $†$ characteristic & Ratio of RRs $\ddagger(95 \% \mathrm{Cl}) ; \mathrm{I} 2 \S$ & P value \\
\hline Attrition $(\geq 10 \% v<10 \%)$ & 1.00 (0.40 to 2.51$) ; 0.61$ & 0.990 \\
\hline Follow-up age (months) $(>6 v \leq 6)$ & 1.23 (0.71 to 2.15$) ; 0.53$ & 0.351 \\
\hline $\begin{array}{l}\text { Maternal postpartum vitamin A supplementation ( } 230 \% \\
\text { mothers) (yes } v \text { no) }\end{array}$ & 1.18 (0.56 to 2.47$) ; 0.60$ & 0.576 \\
\hline No of vitamin A doses received by infant (multiple $v$ single) & 0.80 (0.43 to 1.51$) ; 0.51$ & 0.387 \\
\hline Maternal night blindness ( $\geq 5 \% v<5 \%)$ & 0.67 (0.05 to 9.01$) ; 0.00$ & 0.300 \\
\hline
\end{tabular}

*Multivariate analysis not performed because no study characteristic was significant predictor of response on univariate analysis and number of trials was small.

$\dagger \mathrm{N}=6$ except for maternal night blindness $(n=3)$.

¥For binary variables, estimate of effect size is ratio of relative risks for mortality during infancy (treated/control) in two groups.

§Represents proportion of residual variation caused by heterogeneity. the smaller interactions that are more likely to occur in practice. ${ }^{32}$ We evaluated the stability of our estimate (random effects model) by altering the chosen comparison and control groups in the two relevant factorial designed trials. ${ }^{\text {w14 w15 }}$ On choosing neonatal intervention and control groups irrespective of maternal supplementation status, the sample size of the control component increased in these two trials while the pooled estimate was only marginally lowered $(0.89$, 0.76 to $\left.1.06 ; \mathrm{P}=0.195 ; \mathrm{I}^{2}=49 \%\right)$. When we restricted the analysis to neonatal intervention and control groups with mothers who were either receiving placebo or no supplementation, the sample size of the intervention component diminished in these two trials while the pooled estimates remained almost similar $\left(0.90,0.74,1.10 ; \mathrm{P}=0.303 ; \mathrm{I}^{2}=48 \%\right)$. Thus the conclusion regarding mortality during infancy remained stable irrespective of the chosen comparison and control groups.

\section{Intention to treat analyses}

The Indian trial stated that "all analyses were based on intention to treat." 17 For a "purist" intention to treat analysis, ${ }^{33}$ however, their estimated mortality risk should also have included "infants whose mothers were randomised but who were not enrolled and received supplementation with vitamin A" (see figure 2 of their publication). When we reconstructed such an intention to treat analysis, the relative risk was actually 0.87 ( 0.74 to $1.03 ; \mathrm{P}=0.109$; fixed effects model), which further strengthens our conclusion in this meta-analysis regarding mortality during infancy $(0.94,0.79$ to $1.12 ; \mathrm{P}=0.483 ; \mathrm{I}^{2}=44.7 \%$; random effects model).

\section{Choice of model}

There are no comprehensive rules on when to use random effects or fixed effects models for meta-analysis. A fixed effects model assumes that differences between observed results of trials can be fully accounted for by sampling variation whereas a random effects model assumes that outcomes of trials might differ both because of sampling variation and true diversity in effects. Both models can be appropriately applied for pooling but a random effects model is usually preferred with heterogeneity. We depicted both estimates for entirety, though we preferred the random effects model because we observed substantial heterogeneity $\left(\mathrm{I}^{2}>50 \%\right)$ for mortality during infancy.

No possible at risk groups for evaluating selective supplementation - namely, $\geq 5 \%$ prevalence of maternal night blindness and low birth weight - emerged as significant predictors of mortality but the data were quite limited for a confident interpretation. Appropriately designed trials are therefore required to evaluate survival benefit in these high risk groups, particularly the latter, which contributes substantially to infant mortality. The contradictory findings of an increased risk of acute respiratory infections and a decreased risk of clinic visits are difficult to explain but these pertain to different datasets of two trials each. As earlier reviews in older children have also documented an 
Table 3 | Summary of pooled analyses for all outcomes (relative risks)

\begin{tabular}{|c|c|c|c|c|}
\hline \multirow[b]{2}{*}{ Outcomes } & \multirow[b]{2}{*}{ No of trials } & \multicolumn{2}{|c|}{ RR (95\% Cl); P value } & \multirow{2}{*}{$\begin{array}{l}\text { Heterogeneity I2 (\%); } \\
\text { Q (P value) }\end{array}$} \\
\hline & & Random effects model & Fixed effects model & \\
\hline Mortality during infancy & 6 & 0.92 (0.75 to 1.12$) ; 0.393$ & 0.90 (0.80 to 1.02$) ; 0.090$ & $54.1 ; 10.90(0.053)$ \\
\hline Mortality during neonatal period & 3 & 0.90 (0.75 to 1.08$) ; 0.270$ & 0.90 (0.75 to 1.08$) ; 0.270$ & $0 ; 0.36(0.834)$ \\
\hline \multicolumn{5}{|l|}{ Cause specific mortality: } \\
\hline Respiratory & 4 & 1.11 (0.82 to 1.51$) ; 0.484$ & 1.11 (0.82 to 1.51$) ; 0.484$ & $0 ; 0.34(0.951)$ \\
\hline Diarrhoeal & 4 & 0.98 (0.42 to 2.28$) ; 0.955$ & 0.95 (0.61 to 1.48$) ; 0.809$ & $66 ; 8.83(0.032)$ \\
\hline Others & 4 & 0.76 (0.56 to 1.02$) ; 0.064$ & 0.78 (0.65 to 0.94$) ; 0.009$ & $48.2 ; 5.79(0.122)$ \\
\hline \multicolumn{5}{|l|}{ Morbidities: } \\
\hline Diarrhoea & 3 & 1.04 (0.99 to 1.09$) ; 0.097$ & 1.04 (0.99 to 1.09$) ; 0.097$ & $0 ; 0.99(0.609)$ \\
\hline $\begin{array}{l}\text { Acute respiratory infection or } \\
\text { respiratory difficulty }\end{array}$ & 2 & 1.11 (1.02 to 1.21$) ; 0.015$ & 1.11 (1.02 to 1.21$) ; 0.015$ & $0 ; 0.03(0.867)$ \\
\hline Cough or running nose & 3 & 0.98 (0.85 to 1.13$) ; 0.770$ & 1.03 (0.98 to 1.07$) ; 0.240$ & $68.7 ; 6.4(0.041)$ \\
\hline Ear infection & 1 & 0.33 (0.03 to 3.38); 0.350 & 0.33 (0.03 to 3.38$) ; 0.350$ & Not applicable \\
\hline Fever & 2 & 0.84 (0.48 to 1.47$) ; 0.548$ & 1.02 (0.99 to 1.05$) ; 0.209$ & $61.8 ; 2.62(0.106)$ \\
\hline Vomiting & 1 & 1.22 (0.57 to 2.61$) ; 0.608$ & 1.22 (0.57 to 2.61$) ; 0.608$ & NA \\
\hline \multicolumn{5}{|l|}{ Severe morbidity requiring: } \\
\hline Clinic visits & 2 & 0.81 (0.72 to 0.91$) ; 0.001$ & 0.81 ( 0.72 to 0.91$) ; 0.001$ & $0 ; 0.37(0.542)$ \\
\hline Admission to hospital & 1 & 0.75 (0.26 to 2.16$) ; 0.593$ & 0.75 (0.26 to 2.16$) ; 0.593$ & NA \\
\hline \multicolumn{5}{|l|}{ Early adverse effects: } \\
\hline Bulging fontanelle & 5 & 1.16 (0.81 to 1.65$) ; 0.418$ & 1.06 (0.91 to 1.25$) ; 0.457$ & $65.3 ; 11.52(0.021)$ \\
\hline Vomiting & 4 & 0.92 (0.77 to 1.09 ); 0.308 & 0.91 (0.80 to 1.02$) ; 0.109$ & 23.7; $3.93(0.269)$ \\
\hline Vomiting* & 5 & 0.91 (0.78 to 1.06$) ; 0.214$ & 0.90 (0.80 to 1.02$) ; 0.092$ & $13.9 ; 4.65(0.326)$ \\
\hline Irritability & 3 & 0.98 (0.86 to 1.11$) ; 0.713$ & 0.98 (0.86 to 1.11$) ; 0.700$ & $1.0 ; 2.02(0.364)$ \\
\hline Diarrhoea & 3 & 0.92 (0.64 to 1.33$) ; 0.660$ & 0.93 (0.79 to 1.09 ); 0.358 & $70.1 ; 6.69(0.035)$ \\
\hline Fever & 4 & 0.99 (0.79 to 1.24$) ; 0.916$ & 0.99 (0.79 to 1.24$) ; 0.916$ & $0 ; 1.86(0.603)$ \\
\hline
\end{tabular}

$\mathrm{NA}=$ not applicable.

*If all early adverse effects are presumed to be vomiting in Rahmathullah trial ${ }^{\mathrm{w} 17}$ (see explanation in appendix 1 on bmj.com).

increased risk of acute respiratory tract infections after vitamin A supplementation, ${ }^{34} 35$ this observation might not represent a chance finding.

\section{Comparison with earlier reviews}

Our findings are at variance with a recent review, which states "we identified three reported trials of vitamin A supplementation in the neonatal period in low income countries; they showed a $20 \%$ reduction in mortality in babies younger than 6 months $(\mathrm{RR}=0.80$; 95\% CI 0.66 to 0.96)." ${ }^{12}$ The variation from this earlier estimate could have several explanations. The authors did not explicitly state the inclusion and exclusion criteria, timing of supplementation, choice of control group, and analytical plan to derive their estimate, ${ }^{36}$ which makes direct comparison imprecise. Their comparison group was probably neonatal placebo irrespective of maternal supplementation status, which is different from ours. They also excluded relevant but negative data from three sources. ${ }^{\text {11 w11 w15 }}$ In a subsequent reappraisal after correspondence questioning their finding, ${ }^{13}$ the authors included data from two of the earlier excluded sources. ${ }^{37}$ This pooled estimate also did not document any convincing evidence of a reduction in mortality (relative risk $0.88,0.73$ to 1.06 ; $\mathrm{P}=0.19$ ) after supplementation within three days of birth, which agrees with the results of our meta-analysis. They selectively evaluated reduction in mortality up to age 6 months, ${ }^{37}$ when three trials had follow-up extending to age 1 year. Nevertheless, we did not find any convincing evidence of mortality reduction up to 6 months of age $\left(0.91,0.76\right.$ to $\left.1.09, \mathrm{I}^{2}=43 \%\right)$.

Region specific analyses have suggested evidence of a benefit in south Asia but not in Africa. ${ }^{37}$ We based our subgroup analyses on underlying biological plausibility rather than regional considerations. Also, on metaregression, region was not a significant predictor of heterogeneity $(\mathrm{P}=0.133)$, although the effect sizes seemed disparate $\left(1.13,0.90\right.$ to $1.43, \mathrm{I}^{2}=0 \%$, in Africa and $0.82,0.66$ to $1.02, \mathrm{I}^{2}=45 \%$, in Asia). More trials on neonatal vitamin A supplementation are therefore required to determine if there are true regional differences or some biological characteristics such as high risk populations explain the findings.

We also found no evidence of a reduction in mortality after vitamin A supplementation between 1 and 6 months of age. ${ }^{\text {18-11 }}$ It is difficult to explain the differences from earlier systematic reviews ${ }^{2-4}$ documenting $23-30 \%$ reduction in childhood mortality after intervention after the age of 6 months. There was no evidence of benefit in child survival in a trial conducted recently on one million children in rural India $(0.96$, 0.88 to 1.05$).{ }^{38}$ Trials included in our review were carried out more recently, when the magnitude and severity of vitamin A deficiency in populations might have diminished. Causes of mortality in the neonatal period and in early infancy are different from those after 6 months of age. In high risk settings, a vitamin A 


\section{WHAT IS ALREADY KNOWN ON THIS TOPIC}

Previous systematic reviews have documented that vitamin A supplementation in children over 6 months of age results in $23-30 \%$ reduction in childhood mortality

There is no evidence of reduction in mortality after supplementation between 1 and 6 months

The public health role of neonatal (\&1 month) supplementation in reducing mortality and morbidity in developing countries is controversial

\section{WHAT THIS STUDY ADDS}

There is no convincing evidence of a reduced risk of all cause mortality during infancy or of an increased risk of early adverse effects after neonatal vitamin A supplementation

Risk of neonatal mortality, cause specific mortality, common morbidities, and admission to hospital was not reduced with supplementation, though risk of acute respiratory infection was increased and risk of clinic visits reduced (data from one to four trials)

In developing countries there is no justification for initiating neonatal vitamin A supplementation as a public health intervention for reducing infant mortality and morbidity

deficient state is much more likely after the age of 6 months, when supplementation is more likely to have a beneficial effect.

\section{Predictors of heterogeneity}

We were unable to identify any significant predictor of substantial heterogeneity for mortality. Additional variables, not examined by us, might explain the observed differences. Effects of micronutrient supplementation might be different between boys and girls, possibly because of variations in prevalence of micronutrient deficiency. ${ }^{3940}$ In conformity with the earlier hypothesis, ${ }^{\text {w11 }}$ a post hoc analysis (random effects model in four trials $\left.{ }^{\mathrm{w} 11 \mathrm{w} 12 \mathrm{w} 14 \mathrm{w} 17}\right)$ suggested that the risk of mortality was lower among boys $(0.77,0.59$, $1.01)$ than girls $(0.93,0.73,1.17)$. The two groups, however, were not heterogeneous $(\mathrm{P}=0.400 ; \mathrm{P}=0.439$ for sex on meta-regression). Divergent results might be explained by differences in vaccination intensity because vitamin A supplementation might interact negatively with DPT vaccine in girls. ${ }^{41}$ In the Guinea Bissau trial, ${ }^{\text {w11 }}$ a post hoc analysis suggested that once children received DPT vaccine, mortality in girls who had received vitamin $\mathrm{A}$ at birth was twice that in girls who had received placebo at birth. ${ }^{41}$ We could not explore this hypothesis because of lack of relevant information in the included trials. The possibility of a strong interaction with season in one trial ${ }^{\text {w11 }}$ could not be examined in other trials. None of the trials analysed the relation with infant feeding practices.

\section{Implications for policy}

All the reviewed evidence pertains to developing countries and is primarily from populations at risk of developing vitamin A deficiency. Currently, a public health programme of neonatal synthetic vitamin A supplementation to reduce infant mortality and morbidity is not justified in these settings because there is no convincing evidence of survival benefit or a reduction in morbidity.

\section{Implications for future research}

Future research and trials on neonatal vitamin A supplementation should examine effects on mortality and morbidity in important risk groups (high prevalence of maternal night blindness and low birth weight); mortality in more settings in Asia and Africa to understand regional differences, if any; morbidities and their severity; and relation of mortality to potential predictors of response including exact timing of supplementation, baseline infant mortality rate, maternal vitamin A status, season, sex, vaccination status, and infant feeding practices.

\section{Conclusions}

There is no convincing evidence of a reduced risk of al cause mortality during infancy or of an increased risk of early adverse effects after synthetic neonatal vitamin A supplementation. Limited data do not indicate a reduced risk of mortality during the neonatal period, cause specific mortality, or common morbidities. There is thus no justification for initiating neonatal vitamin A supplementation as a public health intervention in developing countries for reducing infant mortality and morbidity.

We are grateful to Clive Osmond, MRC Epidemiology Resource Centre, University of Southampton, Southampton, for statistical advice. Contributors: SG prepared the protocol, applied the search strategy, retrieved the articles, and extracted data. HSS developed the idea for review, finalised the protocol and search strategy, extracted data, and did the statistical analysis. Both authors wrote the final version of the paper and are guarantors.

Funding: This study was funded by Departments of Child and Adolescent Health and Development, and Nutrition for Health and Development, World Health Organization, Geneva. The funding source had no involvement in the study or the decision to publish the manuscript. Competing interests: None declared.

Ethical approval: Not required.

1 Jones G, Steketee RW, Black RE, Bhutta ZA, Morris SS, Bellagio Child Survival Study Group. How many child deaths can we prevent this year? Lancet 2003;362:65-71.

2 Glasizou PP, Mackerras DEM. Vitamin A supplementation in infectious diseases: a meta-analysis. BMJ 1993;306:366-70.

3 Beaton GH, Martorell R, Aronson KJ, Edmonston B, McCabe G, Ross AC, et al. Effectiveness of vitamin A supplementation in the control of young child morbidity and mortality in developing countries. Geneva: ACC/SCN State of the Art Series, 1993. (Nutrition Policy Discussion Paper No 13.)

4 Fawzi WW, Chalmers TC, Herrera MG, Mosteller F. Vitamin A supplementation and child mortality. JAMA 1993;269:898-903.

5 Rahmathullah L, Underwood BA, Thulsiraj RD, Milton RC, Ramaswamy K, Rahmathullah R, et al. Reduced mortality among children in Southern India receiving a small weekly dose of vitamin A. N Engl J Med 1990;323:929-35.

6 West KP, Pokhrel RP, Katz J, LeClerq S, Khatry SK, Shrestha SR, et al. Efficacy of vitamin A in reducing preschool child mortality in Nepal. Lancet 1991;338:67-71.

7 Ghana VAST Study Team. Vitamin A supplementation in northeastern Ghana: effects on clinic attendances, hospital admissions and child mortality. Lancet 1993;342:7-12.

8 WHO/CHD Immunization-linked Vitamin A Supplementation Study Group. Randomised trial to assess benefits and safety of vitamin A supplementation linked to immunization in early infancy. Lancet 1998;352:1257-63. 
9 Daulaire NMP, Starbuck ES, Houston RM, Church MS, Stukel TA, Pandey MR. Childhood mortality after a dose of vitamin A in a high risk population. BMJ 1992;304:207-10.

10 Newton S, Cousens S, Owusu-Agyei S, Filteau S, Stanley C, Linsell L, et al. Vitamin A supplementation does not affect infants' immune responses to polio and tetanus vaccines. J Nutr 2005;135:2669-73.

11 Newton S, Owusu-Agyei S, Ampofo W, Zandoh C, Adjuik M, Adjei G, et al. Vitamin A supplementation enhances infants' immune responses to hepatitis $B$ vaccine but does not affect responses to Haemophilus influenzae type b vaccine. / Nutr 2007;137:1272-7.

12 Bhutta ZA, Ahmed T, Black RE, Cousens S, Dewey K, Giugliani E, et al, for the Maternal and Child Undernutrition Study Group. What works? Interventions for maternal and child undernutrition and survival. Lancet 2008;371:417-40.

13 Sachdev HPS. Neonatal vitamin A supplementation and infant survival in Asia. Lancet 2008;371:1746.

14 Human development report 2006. New York: United Nations Development Programme. http://hdr.undp.org/en/media/ HDR_2006_Tables.pdf.

15 Darlow BA, Graham PJ. Vitamin A supplementation to prevent mortality and short and long-term morbidity in very low birthweight infants. Cochrane Database Syst Rev 2007;(4):CD000501.

16 Wiysonge CS, Shey MS, Sterne JA, Brocklehurst P. Vitamin A supplementation for reducing the risk of mother-to-child transmission of HIV infection. Cochrane Database Syst ReV 2005;(4):CD003648.

17 Assessment of study quality. In: Higgins JPT, Green S, eds. Cochrane handbook for systematic reviews of interventions 4.2.6. Chichester: John Wiley, 2006 (issue 4, updated Sept 2006).

18 Higgins JPT, Deeks JJ, Altman DG, on behalf of the Cochrane Statistical Methods Group. Section 16.5.4. How to include multiple groups from one study. In: Higgins JPT, Green S, eds. Cochrane handbook for systematic reviews of interventions. Chichester: WileyBlackwell, 2008:510-11.

19 Analyzing and presenting results. In: Higgins JPT, Green S, eds. Cochrane handbook for systematic reviews of interventions 4.2.6. In: Chichester: John Wiley, 2006 (issue 4, updated September 2006).

20 Sterne JAC, Egger M, Smith GD. Investigating and dealing with publication and other biases. In: Egger M, Smith GD, Altman DG, eds. Systematic reviews in health care: meta-analysis in context. London: BMJ Publishing, 2001:189-208.

21 Sterne JAC, Bradburn MJ, Egger M. Meta-analysis in STATA TM. In: Egger M, Smith GD, Altman DG, eds. Systematic reviews in health care: meta-analysis in context. London: BMJ Publishing, 2001:347-69.

22 Steichen TJ, Egger M, Sterne JAC. sbe19.1: tests for publication bias in meta-analysis. Stata Tech Bull 1998;44:3-4.

23 Harris R, Bradburn MJ, Deeks J, Harbord R, Altman D, Steichen T, et al. Stata version 9 update (Distribution Date February 19, 2007) for the Stata User Written Programme sbe24 (Bradburn MJ, Deeks J), Altman D. sbe24: metan—an alternative meta-analysis command. Stata Tech Bull

1999;44:4-15). http://fmwww.bc.edu/RePEc/bocode/m.

24 Higgins JPT, Thompson SG. Quantifying heterogeneity in a metaanalysis. Stat Med 2002;21:1539-58.

25 Harbord R, Steichen T, Sharp SJ. "metareg: update to Stata module to perform meta-analysis regression" (Sharp SJ. sbe23: Meta-analysis regression. Stata Tech Bull

1998;42:16-22). Statistical Software Components S4446201,
Boston College Department of Economics, revised 2 Feb 2005 http://ideas.repec.org/c/boc/bocode/s446201.html.

26 Adamson J, Cockayne S, Puffer S, Torgerson DJ. Review of randomised trials using the post-randomised consent (Zelen's) design. Contemp Clin Trials 2006;27:305-19.

27 Cravioto J. Vitamin A supplementation and child mortalityexamination of a claim. NFI Bull 1990;11:5-6.

28 Gopalan C. Reviews and comments. Child mortality reduction with vitamin A. NFI Bull 1992;13:1-2.

29 Kapil U, Sharma AL. Paradox of vitamin A supplementation to children in India. Indian J Public Health 2005;49:7-10.

30 Tielsch JM. Vitamin A supplements in newborns and child survival. BMJ 2008;336:1385-6.

31 Katz J, West KP Jr, Khatry SK, Pradhan EK, LeClerq SC, Christian P, et al. Maternal low-dose vitamin A or beta-carotene supplementation has no effect on fetal loss and early infant mortality: a randomized cluster trial in Nepal. Am / Clin Nutr 2000;71:1570-6.

32 Brookes ST, Whitely E, Egger M, Davey Smith G, Mulheran PA, Peters TJ. Subgroup analyses in randomized trials: risks of subgroupspecific analyses; power and sample size for the interaction test. J Clin Epidemiol 2004;57:229-36

33 Higgins JPT, Deeks JJ, Altman DG, on behalf of the Cochrane Statistical Methods Group. Intention to treat issues. In: Higgins JPT, Green S, eds. Cochrane handbook for systematic reviews of interventions. Chichester: Wiley-Blackwell, 2008:488-93.

34 Grotto I, Mimouni M, Gdalevich M, Mimouni D. Vitamin A supplementation and childhood morbidity from diarrhea and respiratory infections: a meta-analyis. J Pediatr 2003;142:297-304

35 Gogia S, Sachdev HPS. Web Appendix 10. Review of vitamin A supplementation in pregnancy and childhood. In: Bhutta ZA, Ahmed T, Black RE, Cousens S, Dewey K, Giugliani E, et al, for the Maternal and Child Undernutrition Study Group. What works? Interventions for maternal and child undernutrition and survival. Lancet 2008;371:417-40.

36 Haider BA, Bhutta ZA. Web Appendix 11. Review of vitamin A supplementation in the neonatal period. In: Bhutta ZA, Ahmed T, Black RE, Cousens S, Dewey K, Giugliani E, et al, for the Maternal and Child Undernutrition Study Group. What works? Interventions for maternal and child undernutrition and survival. Lancet 2008;371:417-40.

37 Bhutta ZA, Haider BA, Cousens S, Kirkwood BR, Black RE. Neonatal vitamin A supplementation and infant survival in Asia. Lancet 2008;371:1746-8.

38 Awasthi S, Peto R, Read S, Bundy D. Six-monthly vitamin A from 1 to 6 years of age. DEVTA: cluster randomised trial in 1 million children in North India. www.ctsu.ox.ac.uk/projects/devta/istanbul-vit-alecture.ppt.

39 Wieringa FT, Berger J, Dijkhuizen MA, Hidayat A, Ninh NX, Utomo B, et al. Sex differences in prevalence of anaemia and iron deficiency in infancy in a large multi-country trial in South-East Asia. Br J Nutr 2007;98:1070-6.

40 Webb P, Nishida C, Darnton-Hill I. Age and gender as factors in the distribution of global micronutrient deficiencies. Nutr Rev 2007;65:233-45.

41 Benn CS, Whittle H, Fisker A, Aaby P. Neonatal vitamin A supplementation in South Asia: rapid implementation or understanding the variation? BMJ 2008. www.bmj.com/cgi/eletters/336/7658/1385\#198676.

Accepted: 29 December 2008 